\title{
Dynamical gauge conditions for the Einstein evolution equations
}

\author{
Lee Lindblom and Mark A. Scheel \\ Theoretical Astrophysics 130-33, California Institute of Technology, Pasadena, California 91125, USA
}

(Received 31 January 2003; published 4 June 2003)

\begin{abstract}
The Einstein evolution equations have previously been written in a number of symmetric hyperbolic forms when the gauge fields - the densitized lapse and the shift-are taken to be fixed functions of the coordinates. Extended systems of evolution equations are constructed here by adding the gauge degrees of freedom to the set of dynamical fields, thus forming symmetric hyperbolic systems for the combined evolution of the gravitational and the gauge fields. The associated characteristic speeds can be made causal (i.e., less than or equal to the speed of light) by adjusting 14 free parameters in these new systems, and 21 additional free parameters are available, for example, to optimize the stability of numerical evolutions. The gauge evolution equations in these systems are generalizations of the " $K$-driver" and " $\Gamma$-driver" conditions that have been used with some success in numerical black hole evolutions.
\end{abstract}

DOI: 10.1103/PhysRevD.67.124005

PACS number(s): 04.25.Dm, 02.60.Cb, 04.20.Cv

\section{INTRODUCTION}

The traditional $3+1$ approach to the study of the Einstein evolution equations assumes that spacetime is foliated by a one-parameter family of spacelike surfaces. The spacetime metric is usually decomposed with respect to these $t=$ const surfaces according to

$$
d s^{2}=-N^{2} d t^{2}+g_{i j}\left(d x^{i}+N^{i} d t\right)\left(d x^{j}+N^{j} d t\right),
$$

where $g_{i j}$ is the (positive definite) three-metric on the $t$ $=$ const surfaces, and $N$ and $N^{i}$ are called the lapse and shift, respectively [1]. (The $x^{i}$ represent spatial coordinates on the $t=$ const surfaces.) If $\partial_{t}$ is the tangent vector along which evolutions will be generated, and $\vec{n}=\partial_{\tau}$ is the unit normal to the $t=$ constant surfaces, then the above definitions imply that $\partial_{t}=N \vec{n}+N^{i} \partial_{i}$. Thus the lapse $N$ measures the rate at which proper time $\tau$ advances (as a function of $t$ ) along the unit normals, while the shift $N^{i}$ measures the velocity of points with fixed spatial coordinates with respect to the unit normals.

The lapse $N$ and shift $N^{i}$ are therefore descriptions of how the coordinates $\left\{t, x^{i}\right\}$ are laid out on the spacetime manifold, and so in this sense they represent coordinate or "gauge" degrees of freedom. The lapse and shift are not determined by the Einstein equations, and may be chosen quite freely. For example, the Einstein evolution equations have been written in a variety of symmetric hyperbolic forms in which the (densitized) lapse and shift can be specified as arbitrary functions of the coordinates $\left\{t, x^{i}\right\}$ [2-13].

Since the lapse and shift are not determined by the Einstein equations, we have the opportunity and the responsibility to specify them in some other manner. We may use this freedom in a variety of ways. For example, we could use it to simplify the representation of the spacetime geometry (as is often done in spacetimes with symmetries) [14-16], to simplify the form of the evolution equations [17], to avoid singularities (physical and coordinate) $[1,18,19]$, or to attempt to control the stability of numerical evolutions [20-23].
In this paper we develop systems of evolution equations that include the lapse and the shift as dynamical fields. These equations, together with appropriate versions of the Einstein evolution equations, form symmetric hyperbolic systems for the combined gravitational and gauge fields. Unified hyperbolic systems of equations for the evolution of the gravitational and the gauge fields have been proposed before. The earliest of these uses harmonic gauge conditions that reduce the evolution equations to a very simple form $[17,24]$, but this gauge has not found widespread use in numerical simulations of black hole spacetimes. Dynamical gauge conditions have also been proposed that convert well-known elliptic gauge conditions into equations that are hyperbolic when the other dynamical fields are considered fixed [20,21,25], but these equations have never been fully integrated with the rest of the Einstein evolution equations to form a unified hyperbolic system. Strongly hyperbolic [26] and more recently symmetric-hyperbolic [27] formulations that include rather general evolution equations for the lapse (but which still keep the shift fixed) have also been proposed. Here we propose a new symmetric-hyperbolic system that includes dynamical equations for the lapse and the shift. Our equations are natural generalizations of the " $K$-driver" and the " $\Gamma$-driver" equations that have been used with some success in evolving black hole spacetimes [20-22].

In Sec. II we review the properties of these gauge evolution equations and in Sec. III we combine them with the Einstein evolution equations to form a single unified system. In Sec. IV we show that a 16-parameter family of these combined (gauge and Einstein) evolution equations is symmetric hyperbolic. In Sec. V we find analytical expressions for the characteristic speeds of these new systems. These expressions depend on 14 of the 16 free parameters. We also demonstrate with specific examples that all of these characteristic speeds can be made causal (i.e., less than or equal to the speed of light) by making suitable choices for the 14 parameters. Finally in Sec. VI we extend the evolution equations by performing a general kinematical transformation on the dynamical fields. This transformation depends on 19 additional free parameters which leave the characteristic speeds and the hyperbolicity conditions unchanged. 


\section{DYNAMICAL GAUGE CONDITIONS}

Our aim is to find equations for the gauge fields that allow the spacetime coordinates to adapt dynamically to the structure of the evolving spacetime. In particular, we would like the gauge fields to select coordinates in which all the dynamical fields become time independent whenever the spacetime itself evolves into an equilibrium stationary state. For computational efficiency and ease of formulating appropriate boundary conditions, we prefer to find hyperbolic rather than elliptic equations for the gauge fields. We also prefer hyperbolic equations rather than equations of indeterminant type because they have a well posed initial value problem.

The desire to improve the stability and accuracy of numerical evolutions of Einstein's equations has for many years provided the motivation to find intelligent choices for the gauge fields $[1,18]$. Perhaps the most widely studied gauge condition of this type is the use of maximal-slice foliations for the $t=$ const surfaces in the $3+1$ decomposition. Maximal slices are defined by the condition that the divergence of the normal vector vanishes. Maximal slices tend to avoid strong focusing singularities, and they allow longer numerical evolutions than do simpler choices such as $N$ $=1$. The mathematical condition that a slice be maximal is equivalent to the condition that the trace of the extrinsic curvature of the slice vanishes: $0=K \equiv g^{i j} K_{i j}$. The time evolution of $K$ is determined by the standard 3+1 ArnowittDeser-Misner (ADM) expression

$$
\partial_{t} K-N^{i} \nabla_{i} K=-\nabla^{i} \nabla_{i} N+N K_{i j} K^{i j},
$$

where $\nabla_{i}$ is the covariant derivative compatible with $g_{i j}$. Thus the choice of evolving along a foliation of maximal slices, each with $K=0$, is enforced by imposing an elliptic equation on the lapse $N$. This condition for the lapse is easily generalized to conditions whose effect is to freeze $K$ to its value on an initial surface: $0=\partial_{t} K$. These " $K$-freezing" conditions also result in elliptic equations for the lapse on each time slice:

$$
0=-\partial_{t} K=\nabla^{i} \nabla_{i} N-N K_{i j} K^{i j}-N^{i} \nabla_{i} K
$$

The $K$-freezing conditions have been used numerically with some success [28]. One disadvantage is that they require the solution of an elliptic equation at each time step. This is usually more computationally expensive than solving hyperbolic equations, and for the case of excised black holes $[20,22,28-33]$ it requires appropriate boundary conditions [34] to be imposed on the excision surfaces. For these reasons, alternatives to Eq. (2.2) have been studied as well. One possibility is to convert the elliptic equation for the lapse into a hyperbolic equation by adding suitable time derivative terms. Thus one might take

$$
\partial_{t}^{2} N+\kappa N \partial_{t} N=-\mu N^{2} \partial_{t} K
$$

as a gauge condition [35]. The second time derivative term $\partial_{t}^{2} N$ converts the elliptic equation for $N$ into a hyperbolic equation with characteristic speeds $\pm \sqrt{\mu}$, while the firstorder term $\kappa N \partial_{t} N$ provides dissipation that tends to suppress $\partial_{t} N$. Gauge conditions of this type have been called " $K$-driver" conditions [36] and have been used with some success in the numerical evolution of black hole spacetimes [20,21,23]. A large family of different $K$-driver conditions can be constructed from Eq. (2.3) by adding terms that leave the hyperbolic structure of this equation intact. Here we will use as our starting point one of these $K$-driver equations that admits an exact first time integral. Thus we adopt a firstorder $K$-driver condition which can be thought of as the first integral of an equation like Eq. (2.3):

$$
0=\partial_{t} N-N^{i} \partial_{i} N+\kappa N^{2}+\mu N^{2}\left(K-K_{0}\right) .
$$

Here $K_{0}$ is the arbitrarily prescribed value of $K$ on some $t$ $=$ const surface. Lapse functions that solve this equation will also satisfy a damped wave equation that is analogous to Eq. (2.3). Thus our expectation is that (if and) when a spacetime evolves into a time-independent state, this choice of lapse will drive the evolution toward a slicing in which the trace of the extrinsic curvature $K$ takes the time-independent value $K_{0}$.

Next we turn our attention to finding appropriate conditions for the shift $N^{i}$. The idea is to use our freedom in the shift to select spatial coordinates in which the evolution of the spatial metric $\partial_{t} g_{i j}$ approaches zero whenever the spacetime itself evolves toward a stationary state. The time derivative of the spatial metric is given by the usual $3+1 \mathrm{ADM}$ expression,

$$
\partial_{t} g_{i j}=\nabla_{i} N_{j}+\nabla_{j} N_{i}-2 N K_{i j} \equiv \Sigma_{i j}
$$

York [1] showed that the integral of the square of $\Sigma_{i j}$ $+\bar{\lambda} g_{i j} g^{k l} \Sigma_{k l}$ over a $t=$ const surface is minimized whenever its divergence vanishes:

$$
\begin{aligned}
0= & \nabla_{j}\left(\Sigma^{j i}+\bar{\lambda} g^{j i} \Sigma\right) \\
= & \nabla_{j}\left(\nabla^{j} N^{i}+\nabla^{i} N^{j}+\bar{\lambda} g^{j i} \nabla_{k} N^{k}\right) \\
& -2 \nabla_{j}\left[N\left(K^{j i}+\bar{\lambda} g^{j i} K\right)\right] .
\end{aligned}
$$

This is an elliptic equation for $N^{i}$ whenever $\bar{\lambda}>-2$ [37]. Such a condition selects shift vectors that minimize the time derivative of the spatial metric (or more accurately the time derivative of the densitized metric $g^{\bar{\lambda}} g_{i j}$ ), and includes the well-studied minimal distortion shift condition (the case $\bar{\lambda}$ $\left.=-\frac{1}{3}\right)[1]$.

It would be straightforward to convert the shift conditions of Eq. (2.7) to hyperbolic equations by adding appropriate time derivative terms, in analogy with the derivation of the $K$-driver equation for the lapse. However, we choose instead to follow a slightly different path. Motivated by the work of Alcubierre et al. $[21,28]$ we consider the quantity

$$
\widetilde{\Gamma}^{i} \equiv \tilde{g}^{k l} \widetilde{\Gamma}_{k l}^{i},
$$

where $\tilde{g}_{i j}$ is the conformal metric $\tilde{g}_{i j} \equiv g^{\lambda} g_{i j}, g=\operatorname{det} g_{i j}$, and $\widetilde{\Gamma}^{i}{ }_{k l}$ is the connection compatable with $\widetilde{g}_{i j}$. The quantity $\widetilde{\Gamma}^{i}$ agrees with the dynamical field used in the Baumgarte- 
Shapiro-Shibata and Nakamura (BSSN) $[38,39]$ formulation of the Einstein equations when $\lambda=-\frac{1}{3}$. It follows from Eq. (2.8) that

$$
g^{\lambda} \widetilde{\Gamma}^{i}=-g^{-(1+\lambda) / 2} \partial_{j}\left[g^{(1+\lambda) / 2} g^{j i}\right]
$$

and

$$
\begin{aligned}
\partial_{t}\left(g^{\lambda} \Gamma^{i}\right)= & \partial_{j}\left[\Sigma^{j i}-\frac{1}{2}(1+\lambda) g^{j i} \Sigma\right] \\
& +\frac{1}{2}(1+\lambda)\left[\Sigma^{i j} \partial_{j} \log g+\Sigma \partial_{j} g^{j i}\right] .
\end{aligned}
$$

Thus the " $\Gamma$-freezing" condition $\partial_{t}\left(g^{\lambda} \widetilde{\Gamma}^{i}\right)=0$ imposes an elliptic equation on the shift (for $\lambda<3$ in this case). This $\Gamma$-freezing differential equation has the same principal part as the generalized minimum distortion condition, Eq. (2.6). Following Alcubierre et al. [21] we convert this elliptic shift condition into a hyperbolic equation by adding appropriate time derivative terms, e.g.,

$$
\partial_{t}^{2} N^{i}+\kappa N \partial_{t} N^{i}=\mu N^{2} \partial_{t}\left(g^{\lambda} \widetilde{\Gamma}^{i}\right) .
$$

As was the case for the lapse equation, it is possible to construct a large family of hyperbolic $\Gamma$-freezing conditions by adding non-principal terms to Eq. (2.11). By adding suitable non-principal terms we can construct members of this family that admit exact first integrals. So we adopt as our " $\Gamma$-driver" condition one of these exact first integrals:

$$
0=\partial_{t} N^{i}-N^{j} \partial_{j} N^{i}+\kappa N N^{i}-\mu N^{2}\left(g^{\lambda} \widetilde{\Gamma}^{i}-g_{0}^{\lambda} \widetilde{\Gamma}_{0}^{i}\right) .
$$

Here the time-independent $g_{0}^{\lambda} \widetilde{\Gamma}_{0}^{i}$ is the value of $g^{\lambda} \widetilde{\Gamma}^{i}$ on some particular time slice. Our expectation is that (if and) when a spacetime evolves to a stationary state, that the $\Gamma$-driver condition will cause the spatial coordinates to evolve in a way that tends to minimize the coordinate time derivatives of the spatial metric.

In summary then, we adopt the following $K$-driver and $\Gamma$-driver conditions for the evolution of the lapse and shift:

$$
\begin{aligned}
0= & \partial_{t} N-N^{j} \partial_{j} N+\mu_{L} N^{2}\left(K-K_{0}\right)+\kappa_{L} N^{2}-\epsilon_{L} N \partial_{i} N^{i}, \\
0= & \partial_{t} N^{i}-N^{j} \partial_{j} N^{i}-\mu_{S} N^{2}\left(g^{\lambda} \widetilde{\Gamma}^{i}-g_{0}^{\lambda} \widetilde{\Gamma}_{0}^{i}\right)+\kappa_{S} N N^{i} \\
& -\epsilon_{S} N g^{i j} \partial_{j} N .
\end{aligned}
$$

These conditions are just the $K$-driver and $\Gamma$-driver conditions of Eqs. (2.4) and (2.12), except for the addition of coupling terms between the equations that are proportional to $\epsilon_{L}$ and $\epsilon_{S}$. These coupling terms will give us more flexibility later in constructing a unified system of fully hyperbolic equations for the evolution of all the gravitational and gauge fields. For maximum flexibility, at this stage we take the 7 parameters $\lambda, \mu_{L}, \mu_{S}, \kappa_{L}, \kappa_{S}, \epsilon_{L}$ and $\epsilon_{S}$ to be completely free and undetermined.

\section{UNIFIED EVOLUTION SYSTEM}

The $K$-driver and $\Gamma$-driver Eqs. (2.13) and (2.14) were each constructed to be first-order hyperbolic equations. However, these equations are manifestly hyperbolic only when the other dynamical fields (e.g., $g_{i j}, K_{i j}$ ) are fixed, whereas the situation of interest to us is when all fields evolve together. So our aim now is to construct a unified system of evolution equations for both the gauge and the gravitational fields such that the entire system is symmetric hyperbolic.

The first step is to examine the highest derivative coupling of the (densitized) lapse and shift to the Einstein evolution equations. We use a general form of the equations written in the notation of Kidder-Scheel-Teukolsky (KST) [12]. These are first-order evolution equations for the spatial metric $g_{i j}$, the extrinsic curvature $K_{i j}$ and the spatial derivatives of the metric $D_{k i j}=\frac{1}{2} \partial_{k} g_{i j}$. At this point we need to consider only the highest derivative (or principal) parts of the equations:

$$
\begin{aligned}
\partial_{t} g_{i j} \simeq & N^{n} \partial_{n} g_{i j}+2 g_{n(i} \partial_{j)} N^{n}, \\
\partial_{t} K_{i j} \simeq & N^{n} \partial_{n} K_{i j}-N \partial_{i} \partial_{j} Q-N\left[(1+2 \sigma) g^{c d} \delta^{n}{ }_{(i} \delta^{b}{ }_{j)}\right. \\
& -(1+\zeta) g^{n d} \delta^{b}{ }_{(i} \delta^{c}{ }_{j)}-(1-\zeta) g^{b c} \delta^{n}{ }_{(i} \delta^{d}{ }_{j)} \\
& \left.+g^{n b} \delta^{c}{ }_{i} \delta^{d}{ }_{j}+2 \gamma g^{n[b} g^{d] c} g_{i j}\right] \partial_{n} D_{b c d}, \\
\partial_{t} D_{k i j} \simeq & N^{n} \partial_{n} D_{k i j}+g_{a(i} \partial_{j)} \partial_{k} N^{a}-N\left[\delta^{n}{ }_{k} \delta^{b}{ }_{i} \delta^{c}{ }_{j}\right. \\
& -\frac{1}{2} \eta g^{n b} g_{k(i} \delta^{c}{ }_{j)}-\frac{1}{2} \chi g^{n b} g_{i j} \delta^{c}{ }_{k} \\
& \left.+\frac{1}{2} \eta g^{b c} g_{k(i} \delta^{n}{ }_{j)}+\frac{1}{2} \chi g^{b c} g_{i j} \delta^{n}{ }_{k}\right] \partial_{n} K_{b c},
\end{aligned}
$$

where $\simeq$ denotes equality of the principal part of the equation, and $Q=\log \left(N / g^{\sigma}\right)$ is the densitized lapse. The parameter $\sigma$ that appears in these equations is part of the definition of the densitized lapse $Q$, while $\gamma, \eta, \chi$, and $\zeta$ were introduced by adding multiples of the constraints to the evolution equations (see KST [12]).

The Einstein evolution Eqs. (3.2) and (3.3) couple to the second spatial derivatives of the densitized lapse and shift. Thus in order to construct a first-order unified system, we need to promote the spatial derivatives of the gauge fields to the status of independent dynamical fields; so let

$$
\begin{aligned}
T_{i} & =\partial_{i} Q, \\
M_{k}{ }^{i} & =N^{-1} \partial_{k} N^{i} .
\end{aligned}
$$

Using these definitions we express the gauge evolution equations (2.13) and (2.14) in terms of these new fields. Furthermore, we obtain evolution equations for $T_{i}$ and $M_{k}{ }^{i}$ by taking spatial gradients of Eqs. (2.13) and (2.14). The principal parts of the resulting equations are then given by [40] 


$$
\begin{aligned}
\partial_{t} Q \simeq & 0 \\
\partial_{t} N^{i} \simeq & 0 \\
\partial_{t} T_{i} \simeq & N^{k} \partial_{k} T_{i}+N\left(2 \sigma-\mu_{L}\right) \partial_{i} K+N\left(\epsilon_{L}-2 \sigma\right) \partial_{i} M_{j}{ }^{j}, \\
\partial_{t} M_{j}{ }^{i} \simeq & N^{k} \partial_{k} M_{j}{ }^{i}+2 N \mu_{S} g^{i m} g^{k l} \partial_{j} D_{k l m} \\
& +N\left[2 \epsilon_{S} \sigma-\mu_{S}(1+\lambda)\right] g^{i m} g^{k l} \partial_{j} D_{m k l} \\
& +\epsilon_{S} N g^{i k} \partial_{j} T_{k} .
\end{aligned}
$$

In deriving the last two equations we made use of the constraints

$$
\begin{aligned}
\mathcal{C}_{i j} & \equiv 2 \partial_{[i} T_{j]}=0, \\
\mathcal{C}_{n k}{ }^{i} & \equiv 2 N^{-1} \partial_{[n}\left(N M_{k]}^{i}\right)=0,
\end{aligned}
$$

in order to write all the terms involving $N^{k}$ as advection terms [41].

The system of Eqs. (3.1)-(3.3) and (3.6) -(3.9) constitutes a unified system of first-order evolution equations for the full set of dynamical fields $\left\{g_{i j}, K_{i j}, D_{k i j}, Q, N^{i}, T_{i}, M_{k}{ }^{i}\right\}$ as desired. However, this system is not unique. We are free to add multiples of the various constraints to these equations, thus producing other systems whose constraint-satisfying solutions are identical. Motivated by the fact that the addition of such constraint terms improves the mathematical character of the Einstein evolution equations [12], we now add additional multiples of the constraints to our unified system of equations. In particular, we modify Eqs. (3.3), (3.8), and (3.9) as follows:

$$
\begin{aligned}
\partial_{t} T_{i}= & \ldots+\frac{1}{2} \psi_{1} N \mathcal{C}_{i}+\frac{1}{2} \psi_{2} N \mathcal{C}_{k i}{ }^{k} \\
\partial_{t} M_{j}{ }^{i}= & \cdots+\frac{1}{2} \psi_{3} N g^{i k} \mathcal{C}_{j k}+\frac{1}{2} \psi_{4} N g^{i a} g^{b c} \mathcal{C}_{a j b c} \\
& +\frac{1}{2} \psi_{5} N g^{i b} g^{c a} \mathcal{C}_{a j b c}+\frac{1}{2} \psi_{6} N g^{i a} g^{b c} \mathcal{C}_{a b c j} \\
& +\frac{1}{2} \psi_{7} N \delta_{j}^{i} \mathcal{C}, \\
\partial_{t} D_{k i j}= & +\frac{1}{2} \psi_{8} N g_{a(i)} \mathcal{C}_{j) k}{ }^{a}+\frac{1}{2} \psi_{9} N g_{i j} \mathcal{C}_{k a}{ }^{a} \\
& +\frac{1}{2} \psi_{10} N g_{k(i} \mathcal{C}_{j) a}{ }^{a} .
\end{aligned}
$$

Here the "..." denote the terms in the unmodified equations. The new terms (each proportional to a new constant $\psi_{A}$ ) include multiples of the new constraints, $\mathcal{C}_{i j}$ and $\mathcal{C}_{n k}{ }^{i}$ of Eqs. (3.10) and (3.11), as well as multiples of the standard Hamiltonian and momentum constraints $\mathcal{C}$ and $\mathcal{C}_{i}$, and the constraint $\mathcal{C}_{k l i j}$ from the fixed-gauge Einstein evolution system. These latter constraints are defined by [42]

$$
\begin{aligned}
\mathcal{C} & =\frac{1}{2}\left[{ }^{(3)} R-K_{i j} K^{i j}+K^{2}\right], \\
\mathcal{C}_{i} & =\nabla_{j} K^{j}{ }_{i}-\nabla_{i} K, \\
\mathcal{C}_{k l i j} & =2 \partial_{[k} D_{l] i j} .
\end{aligned}
$$

Adding constraints in this way is essential for obtaining a hyperbolic system of evolution equations. Note that the constraints added here are in addition to the constraints already included in Eqs. (3.2) and (3.3).

The full unified system of evolution equations, including these new constraint terms, can now be written as follows (showing here only the principal parts):

$$
\begin{aligned}
& \partial_{t} g_{i j} \simeq N^{k} \partial_{k} g_{i j}, \\
& \partial_{t} Q \simeq 0, \\
& \partial_{t} N^{i} \simeq 0 \\
& \partial_{t} T_{i} \simeq N^{k} \partial_{k} T_{i}+N\left(2 \sigma-\mu_{L}\right) \partial_{i} K+N\left(\epsilon_{L}-2 \sigma\right) \partial_{i} M_{j}{ }^{j} \\
& +\psi_{1} N \partial_{[k} K_{i]}{ }^{k}+\psi_{2} N \partial_{[k} M_{i]}{ }^{k}, \\
& \partial_{t} M_{j}{ }^{i} \simeq N^{k} \partial_{k} M_{j}{ }^{i}+2 N \mu_{S} g^{i m} g^{k l} \partial_{j} D_{k l m} \\
& +N\left[2 \epsilon_{S} \sigma-\mu_{S}(1+\lambda)\right] g^{i m} g^{k l} \partial_{j} D_{m k l} \\
& +\epsilon_{S} N g^{i k} \partial_{j} T_{k}+\psi_{3} N g^{i k} \partial_{[j} T_{k]} \\
& +N\left[\psi_{4} g^{i[n} \delta_{j}^{a]} g^{b c}+\psi_{5} g^{i(b} g^{c)[n} \delta_{j}^{a]}\right. \\
& \left.+\psi_{6} g^{i[n} g^{a](b} \delta_{j}^{c)}+\psi_{7} g^{a[b} g^{n] c} \delta_{j}^{i}\right] \partial_{n} D_{a b c}, \\
& \partial_{t} D_{k i j} \simeq N^{n} \partial_{n} D_{k i j}+N\left[g_{b(i} \delta_{j)}^{n} \delta_{k}^{a}+\psi_{8} g_{b(i} \delta_{j)}^{[n} \delta_{k}^{a]}\right. \\
& \left.+\psi_{9} g_{i j} \delta_{k}^{[n} \delta_{b}^{a]}+\psi_{10} g_{k(i} \delta_{j)}^{[n} \delta_{b}^{a]}\right] \partial_{n} M_{a}^{b} \\
& -N\left[\delta^{n}{ }_{k} \delta^{b}{ }_{i} \delta^{c}{ }_{j}-\frac{1}{2} \eta g^{n b} g_{k(i} \delta^{c}{ }_{j)}\right. \\
& -\frac{1}{2} \chi g^{n b} g_{i j} \delta_{k}^{c}+\frac{1}{2} \eta g^{b c} g_{k(i} \delta_{j)}^{n} \\
& \left.+\frac{1}{2} \chi g^{b c} g_{i j} \delta_{k}^{n}\right] \partial_{n} K_{b c} . \\
& \partial_{t} K_{i j} \simeq N^{n} \partial_{n} K_{i j}-N \partial_{(i} T_{j)}-N\left[(1+2 \sigma) g^{c d} \delta^{n}{ }_{i} \delta^{b}{ }_{j)}\right. \\
& -(1+\zeta) g^{n d} \delta_{(i}^{b} \delta_{j)}^{c}-(1-\zeta) g^{b c} \delta^{n}{ }_{i} \delta^{d}{ }_{j)} \\
& \left.+g^{n b} \delta^{c}{ }_{i} \delta^{d}{ }_{j}+2 \gamma g^{n[b} g^{d] c} g_{i j}\right] \partial_{n} D_{b c d} \text {. }
\end{aligned}
$$

These equations constitute a first-order system of evolution equations for the dynamical fields $\left\{g_{i j}, K_{i j}, D_{k i j}, Q, T_{i}\right.$, $\left.N^{i}, M_{k}{ }^{i}\right\}$. This system depends on 22 freely specifiable parameters: 20 of these parameters affect the principal parts of the equations $\left\{\sigma, \gamma, \eta, \chi, \zeta, \psi_{1}, \ldots, \psi_{10}, \lambda, \mu_{L}, \mu_{S}\right.$, $\left.\epsilon_{L}, \epsilon_{S}\right\}$, while 2 additional parameters $\left\{\kappa_{L}, \kappa_{S}\right\}$ are dissipation terms in the gauge equations that do not affect the prin- 
cipal parts. We will constrain some of these parameters in the following section to ensure that the system of equations is symmetric hyperbolic. The remaining parameters will be freely specifiable and available for other purposes, such as simplifying the resulting equations or optimizing the stability of numerical spacetime evolutions.

These evolution equations for the dynamical fields $\left\{g_{i j}\right.$, $\left.K_{i j}, D_{k i j}, Q, T_{i}, N^{i}, M_{k}^{i}\right\}$ also imply evolution equations for the various constraints of the system. In Appendix A we derive these constraint evolution equations, and show that if the constraints are exactly satisfied initially then they will continue to be satisfied as the system evolves.

\section{SYMMETRIC HYPERBOLICITY}

The unified system of evolution equations (3.18)-(3.24) derived in Sec. III can be written in the form

$$
\partial_{t} u^{\alpha}+A_{\beta}^{k \alpha} \partial_{k} u^{\beta} \simeq 0,
$$

where $u^{\alpha}$ is the collection of dynamical fields: $u^{\alpha}=\left\{g_{i j}\right.$, $\left.K_{i j}, D_{k i j}, Q, T_{i}, N^{i}, M_{k}{ }^{i}\right\}$. A first-order system such as this is called symmetric hyperbolic if there exists a symmetric positive-definite "symmetrizer" $S_{\alpha \beta}$ on the space of dynamical fields such that $A_{\alpha \beta}^{k} \equiv S_{\alpha \mu} A^{k \mu}{ }_{\beta}$ is symmetric for all $k$ : $A_{\alpha \beta}^{k}=A_{\beta \alpha}^{k}$. Symmetric hyperbolic systems [43] have wellposed initial value problems, real characteristic speeds, complete sets of characteristic eigenvectors, and other nice mathematical properties such as the existence of associated canonical energy norms.

We now explore the conditions under which the unified evolution equations of Sec. III are in fact symmetric hyperbolic. We assume that the symmetrizer $S_{\alpha \beta}$ can be written as a function of the metric $g_{i j}$ and various constant parameters. In particular we consider the following general symmetrizer which we express as a quadratic form [44],

$$
\begin{aligned}
d S^{2} \equiv & S_{\alpha \beta} d u^{\alpha} d u^{\beta} \\
= & A_{1} d G^{2}+A_{2} g^{i k} g^{j l} d \widetilde{g}_{i j} d \widetilde{g}_{k l}+A_{3} d Q^{2}+A_{4} g_{i j} d N^{i} d N^{j}+B_{1} d K^{2}+B_{2} g^{i k} g^{j l} d \widetilde{K}_{i j} d \widetilde{K}_{k l}+C_{1} g^{k l} g^{i a} g^{j b} d \widetilde{D}_{(k i j)} d \widetilde{D}_{(l a b)} \\
& +C_{2} g^{k l} g^{i a} g^{j b}\left[d \widetilde{D}_{k i j}-d \widetilde{D}_{(k i j)}\right]\left[d \widetilde{D}_{l a b}-d \widetilde{D}_{(l a b)}\right]+C_{3} g^{i j} d D_{i}^{1} d D_{j}^{1}+C_{4} g^{i j} d D_{i}^{2} d D_{j}^{2}+2 C_{5} g^{i j} d D_{i}^{1} d D_{j}^{2} \\
& +E_{1} g^{i j} d T_{i} d T_{j}+2 D_{1} g^{i j} d T_{i} d D_{j}^{1}+2 D_{2} g^{i j} d T_{i} d D_{j}^{2}+E_{2} d M^{2}+\frac{1}{2} E_{3}\left[g_{i j} g^{k l}+\delta_{i}^{l} \delta_{j}^{k}\right] d \widetilde{M}_{k}^{i} d \widetilde{M}_{l}^{j} \\
& +\frac{1}{2} E_{4}\left[g_{i j} g^{k l}-\delta_{i}^{l} \delta_{j}^{k}\right] d \widetilde{M}_{k}^{i} d \widetilde{M}_{l}^{j}+2 D_{3} d M d K+2 D_{4} g^{i k} \delta_{l}^{j} d \widetilde{K}_{i j} d \widetilde{M}_{k}^{l} .
\end{aligned}
$$

Here $d G, d K$ and $d M$ are the traces of $d g_{i j}, d K_{i j}$ and $d M_{k}{ }^{i}$, respectively, and $d \widetilde{g}_{i j}, d \widetilde{K}_{i j}$ and $d \tilde{M}_{k}{ }^{i}$ are their tracefree parts. The two traces of $d D_{k i j}$ are defined by

$$
\begin{aligned}
& d D_{i}^{1} \equiv g^{j k} d D_{i j k} \\
& d D_{i}^{2} \equiv g^{j k} d D_{k i j},
\end{aligned}
$$

and its trace-free part, $d \widetilde{D}_{k i j}$, is

$$
\begin{aligned}
d \widetilde{D}_{k i j} \equiv & d D_{k i j}+\frac{1}{5}\left[d D_{(i}^{1} g_{j) k}-2 d D_{k}^{1} g_{i j}+d D_{k}^{2} g_{i j}\right. \\
& \left.-3 d D_{(i}^{2} g_{j) k}\right] .
\end{aligned}
$$

The quadratic form (4.2) is positive definite if and only if the parameters corresponding to diagonal symmetrizer elements $\left\{A_{1}, A_{2}, A_{3}, A_{4}, B_{1}, B_{2}, C_{1}, C_{2}, C_{3}, C_{4}, E_{1}, E_{2}\right.$, $\left.E_{3}, E_{4}\right\}$ are positive, and certain inequalities are satisfied by the parameters corresponding to the off-diagonal symmetrizer elements $\left\{C_{5}, D_{1}, D_{2}, D_{3}, D_{4}\right\}$. Some of these offdiagonal inequalities are simple, i.e., $D_{3}^{2}<E_{2} B_{1}$, and $D_{4}^{2}$ $<B_{2} E_{3}$. But the inequalities involving the other off-diagonal parameters $\left\{C_{5}, D_{1}, D_{2}\right\}$ are less transparent. The needed condition is that the $3 \times 3$ matrix

$$
\left(\begin{array}{ccc}
C_{3} & C_{5} & D_{1} \\
C_{5} & C_{4} & D_{2} \\
D_{1} & D_{2} & E_{1}
\end{array}\right)
$$

is positive definite. The most straightforward way to enforce this condition is to use the fact that a matrix is positive definite if and only if it admits a Cholesky decomposition [45]. By writing the Cholesky decomposition of Eq. (4.6) in terms of new parameters $F_{A}$, we obtain

$$
\begin{aligned}
& C_{3}=F_{1}^{2}+F_{2}^{2}+F_{3}^{2}, \\
& C_{4}=F_{4}^{2}+F_{5}^{2}, \\
& C_{5}=F_{2} F_{4}+F_{3} F_{5}, \\
& D_{1}=F_{3} F_{6}, \\
& D_{2}=F_{5} F_{6}, \\
& E_{1}=F_{6}^{2} .
\end{aligned}
$$


Given these expressions for $\left\{C_{3}, C_{4}, C_{5}, D_{1}, D_{2}, E_{1}\right\}$, the matrix in Eq. (4.6) is positive definite for arbitrary $F_{A}$, so long as $F_{1} \neq 0, F_{4} \neq 0$, and $F_{6} \neq 0$.

It is straightforward (but tedious) now to evaluate the conditions on the various parameters needed to guarantee that the matrices $A_{\alpha \beta}^{k}=S_{\alpha \mu} A^{k \mu}{ }_{\beta}$ are symmetric in $\alpha$ and $\beta$ for all $k$. After lengthy algebraic manipulations we find that the following conditions are necessary and sufficient to guarantee that the $A^{k}{ }_{\alpha \beta}$ are symmetric:

$$
\begin{aligned}
& B_{2}=\left(C_{1}+2 C_{2}\right) / 3 \text {, } \\
& \zeta=-C_{1}\left(E_{3}+D_{4}\right) /\left[E_{3} B_{2}-D_{4}^{2}\right], \\
& \sigma=\left\{3\left(3 D_{3}+2 D_{4}\right)\left[-3\left(2 C_{1}+5 C_{3}+5 C_{4}+10 C_{5}\right) E_{1}+5\left(D_{1}+D_{2}\right)\left(3 D_{1}+3 D_{2}+3 D_{3}+2 D_{4}\right)\right]\right. \\
& -\left(3 E_{2}+2 E_{3}\right)\left\{9 E_{1}\left(2 C_{1}+5 C_{3}+5 C_{4}+10 C_{5}\right)+5\left(D_{1}+D_{2}\right)\left[9\left(B_{1}-D_{1}-D_{2}\right)\right.\right. \\
& \left.\left.\left.+2 C_{1}+4 C_{2}\right]\right\}\right\} /\left[-10 E_{1}\left(3 E_{2}+2 E_{3}\right)\left(9 B_{1}+2 C_{1}+4 C_{2}\right)+30 E_{1}\left(3 D_{3}+2 D_{4}\right)^{2}\right], \\
& \mu_{S}=\left(16 C_{1}+5 C_{2}+15 C_{4}\right) / 30\left(E_{3}+E_{4}\right), \\
& \psi_{8}=2\left(C_{1}-C_{2}+3 D_{4}\right) / 3 C_{2}, \\
& \epsilon_{S}=\left\{5 E_{1}\left(3 E_{2}+2 E_{3}\right)(1-\lambda) \mu_{S}-6 E_{1} C_{1}-15\left(C_{3}+C_{4}+2 C_{5}\right) E_{1}-10 E_{1}\left(3 D_{3}+2 D_{4}\right) \sigma\right. \\
& \left.+5\left(D_{1}+D_{2}\right)\left[3\left(D_{1}+D_{2}+D_{3}\right)+2 D_{4}\right]\right\} / 5\left(3 E_{2}+2 E_{3}\right)\left(D_{1}+D_{2}-2 \sigma E_{1}\right), \\
& \mu_{L}=2 \sigma+\left[3 B_{1}+2 B_{2}-3\left(D_{1}+D_{2}\right)-\left(3 D_{3}+2 D_{4}\right) \epsilon_{S}\right] / 3 E_{1}, \\
& \epsilon_{L}=2 \sigma-\left[3\left(D_{1}+D_{2}+D_{3}\right)+2 D_{4}-\left(3 E_{2}+2 E_{3}\right) \epsilon_{S}\right] / 3 E_{1}, \\
& \psi_{3}=-\left(1+E_{3} / E_{4}\right) \epsilon_{S}+\left(D_{2}+D_{4}\right) / E_{4}, \\
& \psi_{4}=\left\{2 C_{1}-5 C_{2}-15\left[C_{5}+(1+2 \sigma) D_{4}\right]-15\left(E_{3}+E_{4}\right)\left[(1+\lambda) \mu_{S}-2 \sigma \epsilon_{S}\right]\right\} / 15 E_{4}, \\
& \psi_{5}=\left(C_{1}+2 C_{2}+3 D_{4}\right) / 3 E_{4}+\left(C_{1}-\zeta D_{4}\right) / E_{3}, \\
& \psi_{6}=\left(C_{1}+2 C_{2}+3 D_{4}\right) / 3 E_{4}-\left(C_{1}-\zeta D_{4}\right) / E_{3}, \\
& \gamma=\left\{\left[3 C_{1}-15\left(C_{3}+C_{5}\right)+30 \sigma D_{1}\right]\left(D_{3}+E_{2}\right)+5\left(6 B_{1}+B_{2}\right) E_{2}-5\left(6 D_{3}+D_{4}+3 \epsilon_{L} D_{1}\right) D_{3}\right. \\
& -15 \mu_{L} D_{1} E_{2}+10\left(D_{4} E_{2}-D_{3} E_{3}\right)(1+\lambda) \mu_{S}-10 \sigma\left(3 D_{3}+2 D_{4}\right) D_{3}+20 \sigma \epsilon_{S}\left(D_{3} E_{3}-D_{4} E_{2}\right) \\
& \left.+10 \sigma\left(3 B_{1}+2 B_{2}\right) E_{2}\right\} / 45\left(D_{3}^{2}-B_{1} E_{2}\right) \text {, } \\
& \chi=\left\{\left[2\left(C_{3}+2 C_{5}\right) E_{1}-2\left(D_{1}+2 D_{2}\right) D_{1}\right]\left[30 \mu_{S} D_{4} E_{1}+\left(16 C_{1}+5 C_{2}+15 C_{4}\right) E_{1}+15\left(B_{2}-D_{2}-\epsilon_{S} D_{4}\right) D_{2}\right]\right. \\
& +\left[\left(2 C_{4}+C_{5}\right) E_{1}-\left(D_{1}+2 D_{2}\right) D_{2}\right]\left[30\left(D_{2}+\epsilon_{S} D_{4}\right) D_{1}-2\left(8 C_{1}-5 C_{2}+15 C_{5}\right) E_{1}\right. \\
& \left.\left.+15 D_{4} E_{1}\left(2 \mu_{S}+2 \lambda \mu_{S}-\psi_{5}+\psi_{6}-4 \sigma \epsilon_{S}\right)-30 B_{2}\left(D_{1}-2 \sigma E_{1}+\zeta E_{1}\right)\right]\right\} / 75 E_{1}\left[C_{3} D_{2}^{2}+C_{5}^{2} E_{1}\right. \\
& \left.-2 C_{5} D_{1} D_{2}+\left(D_{1}^{2}-C_{3} E_{1}\right) C_{4}\right] \text {, } \\
& \eta=\left[15(2-\chi) D_{2}^{2}+15\left(2 \epsilon_{S} D_{4}-3 \chi D_{1}\right) D_{2}+8 C_{1} E_{1}+10 C_{2} E_{1}+15\left(\psi_{5}-\psi_{6}-4 \mu_{S}\right) D_{4} E_{1}+15(\chi-2) C_{4} E_{1}\right. \\
& \left.-30\left(D_{2}+E_{1}-\zeta E_{1}\right) B_{2}+45 \chi C_{5} E_{1}\right] / 15\left[\left(D_{1}+2 D_{2}\right) D_{2}-\left(2 C_{4}+C_{5}\right) E_{1}\right], \\
& \psi_{2}=\left\{-16 C_{1}\left(C_{4} D_{1}+2 C_{3} D_{2}-2 C_{5} D_{1}-C_{5} D_{2}\right)-5 C_{2}\left[C_{4} D_{1}+C_{5}\left(D_{1}-D_{2}\right)-C_{3} D_{2}\right]\left(4+3 \psi_{8}\right)\right. \\
& +30\left(C_{3} C_{4}-C_{5}^{2}\right)\left[D_{4}+\left(E_{4}-E_{3}\right) \epsilon_{S}+E_{4} \psi_{3}\right]+60\left(C_{3} D_{2}-C_{5} D_{1}\right)\left(E_{3}-E_{4}\right) \mu_{S} \\
& \left.-\left(C_{4} D_{1}-C_{5} D_{2}\right) \Upsilon\right\} / 30\left[E_{1}\left(C_{5}^{2}-C_{3} C_{4}\right)+C_{3} D_{2}^{2}+C_{4} D_{1}^{2}-2 C_{5} D_{1} D_{2}\right], \\
& \psi_{7}=\left[2 C_{1}-5 C_{2}+15 C_{4}+30 C_{5}-60 D_{3}-90 \gamma D_{3}-10 D_{4}-10\left(E_{3}+6 E_{2}-3 E_{4}\right) \mu_{S}+30\left(\epsilon_{L}-2 \sigma\right) D_{2}\right] / 45 E_{2}, \\
& \psi_{1}=\left[-2 B_{2}-(3 \chi+\eta) D_{1}+(2-\chi-2 \eta) D_{2}+2 D_{4} \epsilon_{S}\right] / E_{1},
\end{aligned}
$$




$$
\begin{aligned}
\psi_{10}= & \left\{\left(3 C_{3}+C_{5}\right)\left[60\left(E_{4}-E_{3}\right) \mu_{S}-5 C_{2}\left(4+3 \psi_{8}\right)+32 C_{1}+30 D_{2} \psi_{2}-15 C_{4} \psi_{8}+30 C_{5}\left(2+\psi_{8}\right)\right]\right. \\
& \left.-\left(C_{4}+3 C_{5}\right)\left[16 C_{1}+5 C_{2}\left(4+3 \psi_{8}\right)+30 D_{1} \psi_{2}-15 C_{5} \psi_{8}+30 C_{3}\left(2+\psi_{8}\right)+\Upsilon\right]\right\} / 150\left(C_{3} C_{4}-C_{5}^{2}\right), \\
\psi_{9}= & {\left[32 C_{1}-5\left(4+3 \psi_{8}\right) C_{2}-15\left(\psi_{8}+4 \psi_{10}\right) C_{4}+30\left(2+\psi_{8}-\psi_{10}\right) C_{5}+60\left(E_{4}-E_{3}\right) \mu_{S}+30 \psi_{2} D_{2}\right] / 30\left(C_{4}+3 C_{5}\right), }
\end{aligned}
$$

where $Y$ is given by

$$
\begin{aligned}
\Upsilon= & 30(1+\lambda)\left(E_{3}-E_{4}\right) \mu_{S}+30 D_{4}(2 \sigma-\zeta)-15 E_{4}\left(2 \psi_{4}+\psi_{5}\right. \\
& \left.+\psi_{6}-4 \sigma \epsilon_{S}\right)+15 E_{3}\left(\psi_{6}-\psi_{5}-4 \sigma \epsilon_{S}\right) .
\end{aligned}
$$

These conditions determine the 20 parameters $\left\{B_{2}\right.$, $\left.\psi_{1}, \ldots, \psi_{10}, \sigma, \gamma, \eta, \chi, \zeta, \mu_{L}, \mu_{S}, \epsilon_{L}, \epsilon_{S}\right\}$ in terms of the 15 parameters $\left\{\lambda, B_{1}, C_{1}, C_{2}, C_{3}, C_{4}, C_{5}, D_{1}, D_{2}\right.$, $\left.D_{3}, D_{4}, E_{1}, E_{2}, E_{3}, E_{4}\right\}$ [46]. Writing the conditions for symmetric hyperbolicity like this is a particularly convenient way to parametrize these evolution systems. The parameters $\left\{\lambda, B_{1}, C_{1}, C_{2}, C_{3}, C_{4}, C_{5}, D_{1}, D_{2}, D_{3}, D_{4}, E_{1}, E_{2}\right.$, $\left.E_{3}, E_{4}\right\}$ can be chosen freely except for the simple inequalities needed to guarantee the positivity of $S_{\alpha \beta}$. We note that the evolution system is invariant under an overall scaling of the symmetrizer. Thus without loss of generality we will set $C_{1}=1$, so there are really only 14 freely specifiable parameters that affect the evolution equations. We also point out the following nice feature of this way of parametrizing these equations: By using the symmetrization conditions in Eqs. (4.13)-(4.33) to determine the parameters that actually appear in the evolution equations $\left\{\psi_{1}, \ldots, \psi_{10}, \sigma, \gamma, \eta, \chi\right.$, $\left.\zeta, \mu_{L}, \mu_{S}, \epsilon_{L}, \epsilon_{S}\right\}$ we are guaranteed to have a system that has only real characteristic speeds, a complete set of eigenvectors, etc. This same parametrization technique has been used by Frittelli and Reula [9], and can also be used to provide a more convenient and complete characterization of the symmetric hyperbolic subset of the original fixed-gauge KST equations. We summarize this approach to the KST equations in Appendix B. Finally we note that the four symmetrizer parameters $\left\{A_{1}, A_{2}, A_{3}, A_{4}\right\}$ do not enter any of the symmetry conditions. So while these parameters can be chosen quite freely (ensuring only that they are positive) they do not seem to play any important role in determining the dynamics of the system.

\section{CHARACTERISTIC SPEEDS}

The evolution equations for the full system of fieldsincluding the gauge fields-have been put in a first-order form in Sec. III. The characteristic speeds in the direction $\xi_{k}$ are defined as the eigenvalues of the matrix $\xi_{k} A^{k \alpha}{ }_{\beta}$ that appears in Eq. (4.1). The unit one-form $\xi_{k}$ specifies the direction of propagation. The characteristic speeds associated with the fields $\left\{g_{i j}, Q, N^{i}\right\}$ are very simple. In the frame of the hypersurface-normal observers, the characteristic speed associated with the propagation of $g_{i j}$ is $v=0$, while the speed associated with the propagation of the gauge fields $\left\{Q, N^{i}\right\}$ is $v=-\xi_{k} N^{k} / N[47]$.

In order to evaluate the characteristic speeds associated with the other dynamical fields of this system it is convenient to transform to an irreducable representation of the space of fields. In this basis the matrix $\xi_{k} A^{k \alpha}{ }_{\beta}$ becomes block diagonal and hence its eigenvalues become much easier to evaluate. The irreducable representation of the remaining dynamical fields $\left\{T_{i}, M_{k}{ }^{i}, K_{i j}, D_{k i j}\right\}$ consists of projecting them onto the scalars $\left\{T_{i} \xi^{i}, M_{k}{ }^{i} \xi^{k} \xi_{i}, M_{k}{ }^{i} P^{k}{ }_{i}, K_{i j} \xi^{i} \xi^{j}, K_{i j} P^{i j}\right.$, $\left.D_{k i j} \xi^{k} \xi^{i} \xi^{j}, D_{k i j} \xi^{k} P^{i j}, D_{k i j} P^{k i} \xi^{j}\right\}$ (where $P_{i j}=g_{i j}-\xi_{i} \xi_{j}$ is the projection tensor onto the two-space orthogonal to $\xi_{i}$ ), the transverse vectors $\left\{T_{i} P^{i}{ }_{j}, \quad M_{k}{ }^{i} \xi^{k} P_{i}{ }^{j}, \quad M_{k}{ }^{i} \xi_{i} P^{k j}\right.$, $K_{i k} \xi^{i} P^{k j}, \quad D_{k i l} \xi^{k} \xi^{i} P^{l j}, \quad D_{k i l} P^{k j} \xi^{i} \xi^{l}, \quad D_{k i l} P^{k(i} P^{l) j}$, $\left.D_{k i l} P^{k j} P^{i l}\right\}$, the symmetric transverse traceless tensors $\left\{M_{k l} P^{k l}{ }_{i j}, K_{k l} P^{k l}{ }_{i j}, D_{m k l} \xi^{m} P^{k l}{ }_{i j}, D_{m k l} \xi^{l} P^{m k}{ }_{i j}\right\}$ (where $P^{k l}{ }_{i j}=P^{k}{ }_{i} P_{j}^{l}-\frac{1}{2} P^{k l} P_{i j}$ ), the antisymmetric transverse tensors $\left\{M_{k}^{l} P^{k}{ }_{[i} P_{j] l}, K_{k l} P^{k}{ }_{[i} P_{j]}^{l}\right\}$, and finally the transverse traceless part of $D_{k i j}$.

The scalar parts of the dynamical fields form an 8 -dimensional subspace, and this $8 \times 8$ block of $\xi_{k} A^{k \alpha}{ }_{\beta}$ decouples from the others. This block depends on the dynamical KST parameters and the other parameters introduced in Sec. III that describe the dynamics of the gauge fields. We find that the eight characteristic speeds (relative to the normals of the hypersurface) can be represented as

$$
\begin{aligned}
& v_{S 1 \pm}^{2}=A_{S 1} \pm B_{S 1}, \\
& v_{S 2 \pm}^{2}=A_{S 2} \pm B_{S 2},
\end{aligned}
$$

where

$$
\begin{aligned}
A_{S 1}= & \frac{1}{2}\left[\mu_{L}+(1-\lambda) \mu_{S}+\epsilon_{S} \epsilon_{L}\right], \\
B_{S 1}^{2}= & A_{S 1}^{2}+(1-\lambda)\left(\epsilon_{L}-\mu_{L}\right) \mu_{S}, \\
A_{S 2}= & \frac{1}{4}[(1+2 \gamma)(2+2 \chi-\eta)-\eta \zeta] \\
& +\frac{1}{16}\left(2+\psi_{8}+2 \psi_{10}\right)\left(\psi_{5}-\psi_{6}\right) \\
& +\frac{1}{8} \psi_{7}\left(2+3 \psi_{8}-4 \psi_{9}+2 \psi_{10}\right),
\end{aligned}
$$




$$
\begin{aligned}
B_{S 2}^{2}= & A_{S 2}^{2}-\frac{1}{8}\left[(1+\chi)\left(2+\psi_{8}+2 \psi_{10}\right)\right. \\
& \left.+\eta\left(\psi_{8}-2 \psi_{9}\right)\right]\left[(1+2 \gamma)\left(\psi_{5}-\psi_{6}\right)-2 \zeta \psi_{7}\right] .
\end{aligned}
$$

The transverse vector parts of the dynamical fields constitute two identical 8-dimensional subspaces. The eight characteristic speeds for each of these blocks are

$$
\begin{gathered}
v_{V 0}^{2}=0, \\
v_{V 1}^{2}=\mu_{S}, \\
v_{V 2 \pm}^{2}=A_{V 2} \pm B_{V 2},
\end{gathered}
$$

where

$$
\begin{aligned}
A_{V 2}= & -\frac{1}{8}\left(\psi_{1}+\psi_{2} \psi_{3}\right)+\frac{1}{8} \psi_{4}\left(2+\psi_{8}-3 \psi_{9}-\psi_{10}\right) \\
& +\frac{1}{8} \psi_{5}\left(2+\psi_{8}-\psi_{9}\right)-\frac{1}{16} \psi_{6}\left(2 \psi_{9}+3 \psi_{10}\right) \\
& +\frac{1}{16} \eta(1-3 \zeta-4 \sigma)-\frac{1}{8} \chi(1+6 \sigma), \\
B_{V 2}^{2}= & A_{V 2}^{2}+\chi \Lambda_{\chi}+\eta \Lambda_{\eta}+\Lambda, \\
\Lambda_{\chi}= & \frac{1}{16}\left(2+\psi_{8}\right)\left[(1+3 \zeta) \psi_{4}+(1+4 \sigma+\zeta) \psi_{5}\right. \\
& \left.-(2 \sigma-\zeta) \psi_{6}\right]-\frac{1}{32} \psi_{10}\left[(5-9 \zeta) \psi_{4}\right. \\
& \left.+(1-4 \sigma-3 \zeta) \psi_{5}+(4+14 \sigma-3 \zeta) \psi_{6}\right] \\
& -\frac{1}{16} \psi_{2}\left[(1+6 \sigma) \psi_{3}+3 \psi_{4}+\psi_{5}+\psi_{6}\right] \\
\Lambda_{\eta}= & \frac{1}{32} \psi_{2}\left[(1-4 \sigma-3 \zeta) \psi_{3}-2 \psi_{4}-3 \psi_{6}\right] \\
& +\frac{1}{32}\left(2+\psi_{8}\right)\left[3(\zeta-2 \sigma) \psi_{6}-(1-4 \sigma-3 \zeta) \psi_{5}\right. \\
& \left.+(5 \zeta-1) \psi_{4}\right]+\frac{1}{32} \psi_{9}\left[(1-4 \sigma-3 \zeta) \psi_{5}\right. \\
& \left.+(5-9 \zeta) \psi_{4}+(4+14 \sigma-3 \zeta) \psi_{6}\right] \\
& \frac{1}{16} \psi_{10}\left[(1-4 \sigma-3 \zeta) \psi_{3}-2 \psi_{4}-3 \psi_{6}\left[\psi_{5}+(1+6 \sigma) \psi_{3}+3 \psi_{4}+\psi_{6}\right]\right.
\end{aligned}
$$

We note that the parameter $\mu_{S}$ introduced in Eq. (2.14) represents one of the characteristic speeds of this system, as expected.

The characteristic speeds of the two identical 4-dimensional spaces of symmetric transverse traceless second rank tensors are

$$
\begin{aligned}
& v_{(T T) 1}^{2}=1, \\
& v_{(T T) 2}^{2}=\frac{1}{8}\left(\psi_{5}-\psi_{6}\right)\left(2+\psi_{8}\right) .
\end{aligned}
$$

The characteristic speeds of the 2-dimensional space of antisymmetric transverse-traceless second-rank tensors are

$$
v_{[T T]}^{2}=\frac{1}{8}\left(\psi_{5}+\psi_{6}\right)\left(2+\psi_{8}\right) .
$$

And finally the subspace consisting of the transverse traceless part of $D_{k i j}$ has only one characteristic speed, and this vanishes.

These expressions determine the characteristic speeds in terms of the parameters $\left\{\psi_{1}, \psi_{2}, \ldots\right\}$ that define the form of the evolution equations. The speeds can also be re-expressed in terms of the symmetrizer parameters through Eqs. (4.13)(4.33). The characteristic speeds are therefore functions of the 14 parameters $\left\{B_{1}, C_{2}, C_{3}, C_{4}, C_{5}, D_{1}, D_{2}, D_{3}, D_{4}\right.$, $\left.E_{1}, E_{2}, E_{3}, E_{4}, \lambda\right\}$ that can be specified (almost) freely as discussed in Sec. IV.

Although most of the characteristic speeds depend on the parameters that define the system of evolution equations, several of the speeds are independent of them. For instance, 12 eigenvectors have characteristic speed zero, and four eigenvectors have characteristic speed \pm 1 (the speed of light in our units); the former correspond to gauge-dependent fields, while the latter must be the incoming and outgoing fields corresponding to the two physical gravitational degrees of freedom. The remaining speeds, the adjustable ones, must correspond to various gauge-dependent and therefore basically unphysical characteristic fields.

In the past it has been considered most natural $[7,12,48]$ to set any adjustable speeds in the Einstein evolution equations to one (the speed of light) or zero with respect to the $t=$ const surface normals. Our experience, however, is that the instabilities limiting evolutions of black hole spacetimes (with excision) often occur in outgoing characteristic fields that propagate at the speed of light just outside the event horizon [13]. Excitations in such fields remain in the computational domain for long periods of time and therefore have the opportunity to grow large. It therefore might be better to set the adjustable characteristic speeds to values significantly less than the speed of light for evolutions of black hole spacetimes.

We have not been able to show that the adjustable characteristic speeds can be set to arbitrary values by adjusting the available parameters, and in fact it appears likely that this is not possible. In particular we have not been able to find parameter values that make all of these speeds equal to unity or zero. However, we have shown that parameter values can 
be chosen to make all of the adjustable characteristic speeds causal (i.e. less than or equal to the speed of light) [49]. To provide a specific example, we have found parameter values that make the characteristic speeds take the following "simple" values,

$$
\begin{aligned}
& 0=v_{(T T) 2}^{2}=v_{[T T]}^{2}, \\
& \frac{1}{4}=v_{S 1-}^{2}=v_{S 2-}^{2}=v_{V 2-}^{2}, \\
& \frac{1}{2}=v_{S 1+}^{2}=v_{S 2+}^{2}=v_{V 1}^{2}=v_{V 2+}^{2},
\end{aligned}
$$

to any desired accuracy. The approximate values of the symmetrizer parameters needed to achieve these characteristic speeds are as follows: $B_{1}=7.17, C_{1}=1.00, C_{2}=2.57, C_{3}$ $=8.68, C_{4}=3.95, C_{5}=-3.81, D_{1}=5.36, D_{2}=4.86, D_{3}=$ $-10.78, D_{4}=-2.04, E_{1}=44.64, E_{2}=19.39, E_{3}=3.65, E_{4}$ $=2.22, \lambda=-0.33$. These symmetrizer parameters also determine the parameters that define the explicit form of the evolution equations; for this example the latter parameters have the following approximate values: $\psi_{1}=0.13, \psi_{2}=$ $-0.34, \quad \psi_{3}=4.54, \quad \psi_{4}=-0.92, \quad \psi_{5}=0.00, \quad \psi_{6}=0.00, \quad \psi_{7}=$ $-0.90, \psi_{8}=-2.00, \psi_{9}=-0.23, \psi_{10}=0.27, \gamma=-0.76, \sigma$ $=0.50, \quad \zeta=-0.49, \quad \eta=0.93, \quad \chi=-0.43, \quad \mu_{S}=0.50, \quad \mu_{L}$ $=0.63, \epsilon_{S}=-1.24, \epsilon_{L}=0.44$. We see that all of the characteristic speeds in this example are causal, and the various parameters that determine the evolution equations are all of order unity. In another example, we explored the possibility of making all of the characteristic speeds which appear in Eqs. (5.18)-(5.20) as small as possible. We found that it was only possible to make the squares of all these characteristic speeds smaller than about 0.29 . Thus it is relatively easy to find examples of these evolution equations that appear to be reasonable candidates for performing numerical evolutions of black hole spacetimes.

\section{KINEMATICAL EXTENSION}

Finally we note that the independent dynamical fields $u^{\alpha}$ $\equiv\left\{g_{i j}, K_{i j}, D_{k i j}, Q, T_{i}, N^{i}, M_{k}{ }^{i}\right\}$ can also be modified in these evolution equations. It has been shown $[12,13,32]$ that seemingly trivial changes in the choice of these dynamical fields can have dramatic effects on the stability of numerical spacetime evolutions. So following KST [12] we introduce a set of linear transformations on the dynamical fields. In particular we take a new set of fields $\hat{u}^{\alpha}$ defined by a transformation of the form $\hat{u}^{\alpha}=T^{\alpha}{ }_{\beta} u^{\beta}$, where $T^{\alpha}{ }_{\beta}$ depends only on various parameters and the spatial metric $g_{i j}$. The most general such transformation (which preserves the fundamental metric fields $\left.\left\{g_{i j}, Q, N^{i}\right\}\right)$ is [50]

$$
\hat{K}_{i j}=K_{i j}+\hat{z}_{1} g_{i j} g^{a b} K_{a b}+\hat{z}_{2} M_{(i}{ }^{a} g_{j) a}+\hat{z}_{3} g_{i j} M_{a}^{a}
$$

$$
\begin{aligned}
\hat{M}_{k}{ }^{i}= & \hat{z}_{4} M_{k}{ }^{i}+\hat{z}_{5} \delta_{k}^{i} M_{a}^{a}+\frac{1}{2} \hat{z}_{6}\left(\delta_{k}^{a} \delta_{b}^{i}-g^{i a} g_{k b}\right) M_{a}^{b} \\
& +\hat{z}_{7} K_{k a} g^{a i}+\hat{z}_{8} \delta_{k}^{i} g^{a b} K_{a b} \\
\hat{D}_{k i j}= & {\left[\hat{k}_{1} \delta_{k}^{a} \delta_{i}^{b} \delta_{j}^{c}+\hat{k}_{2} \delta_{(i}^{a} \delta_{j)}^{b} \delta_{k}^{c}+\hat{k}_{3} g_{i j} g^{b c} \delta_{k}^{a}\right.} \\
& \left.+\hat{k}_{4} g_{i j} g^{a b} \delta_{k}^{c}+\hat{k}_{5} g_{k(i} \delta_{j)}^{a} g^{b c}+\hat{k}_{6} g_{k(i} \delta_{j)}^{c} g^{a b}\right] D_{a b c} \\
& +\hat{k}_{7} T_{k} g_{i j}+\hat{k}_{8} g_{k(i} T_{j)}, \\
\hat{T}_{i}= & \hat{k}_{9} T_{i}+\hat{k}_{10} g^{a b} D_{i a b}+\hat{k}_{11} g^{a b} D_{a b i} .
\end{aligned}
$$

This transformation is (generically) invertable, and is the identity when $\hat{z}_{4}=\hat{k}_{1}=\hat{k}_{9}=1$ and all other $\hat{z}_{A}$ and $\hat{k}_{A}$ vanish. Note that as in KST [12], it is understood that when constructing evolution equations for the transformed fields $\hat{u}^{\alpha}$, all (temporal and spatial) derivatives of $g_{i j}$ that are introduced by differentiating $T^{\alpha}{ }_{\beta}$ are to be eliminated by substituting the definition of $D_{k i j}$ and the evolution equations for $g_{i j}$.

We also note that the kinematical transformations $\hat{u}^{\alpha}$ $=T^{\alpha}{ }_{\beta} u^{\beta}$ described by Eqs. (6.1)-(6.4) do not change the hyperbolicity of the system or the characteristic speeds. This is because the characteristic matrix for the transformed system is $\xi_{k} \hat{A}^{k \alpha}{ }_{\beta} \equiv \xi_{k} T^{\alpha}{ }_{\mu} A^{k \mu}{ }_{\nu}\left(T^{-1}\right)^{\nu}{ }_{\beta}$, which has the same eigenvalues as $\xi_{k} A_{\beta}^{k \alpha}$, and the symmetrizer for the transformed system is $\hat{S}_{\alpha \beta} \equiv S_{\mu \nu}\left(T^{-1}\right)^{\mu}{ }_{\alpha}\left(T^{-1}\right)^{\nu}{ }_{\beta}$, which is symmetric and positive definite iff $S_{\mu \nu}$ is symmetric and positive definite.

In summary, the unified system of evolution equations presented here contains 41 free parameters when written in terms of the dynamical fields $\hat{u}^{\alpha}$ : the 22 parameters that entered Eqs. (3.18)-(3.24) as described above, plus 19 transformation parameters $\left\{\hat{z}_{1}, \ldots, \hat{z}_{8}, \hat{k}_{1}, \ldots, \hat{k}_{11}\right\}$. In Sec. IV we reduced the number of free parameters by 6 to ensure that the system of equations is symmetric hyperbolic. The remaining 35 parameters are freely specifiable and available for other purposes, such as simplifying the resulting equations, fixing the characteristic speeds to desired values, or optimizing the stability of numerical spacetime evolutions.

\section{ACKNOWLEDGMENTS}

We thank Michael Holst, Markus Keel, Lawrence Kidder, Harald Pfeiffer, and Manuel Tiglio for helpful conversations concerning this work. This research was supported in part by NSF grant PHY-0099568 and NASA grants NAG5-10707 and NAG5-12834.

\section{APPENDIX A: CONSTRAINT EVOLUTION}

The dynamical system derived in Sec. III represents the Einstein evolution equations only when certain constraints are satisfied. Here we derive the system of evolution equations that the constraints themselves satisfy. We begin by giving explicit expressions for each of the constraints in terms of the dynamical fields and their spatial derivatives: 


$$
\begin{aligned}
\mathcal{C}= & \left(g^{i k} g^{j l}-g^{i j} g^{k l}\right) \partial_{l} D_{k i j}+g^{i[j} g^{a] b} K_{i j} K_{a b} \\
& -\frac{1}{2}\left(g^{k c} g^{i j} g^{a b}+2 g^{k a} g^{i b} g^{j c}+8 g^{k[i} g^{a] j} g^{c b}\right. \\
& \left.-3 g^{k c} g^{i a} g^{j b}\right) D_{k i j} D_{c a b}, \\
\mathcal{C}_{i}= & \left(g^{a j} g^{b k} \delta_{i}^{c}+g^{c j} g^{a b} \delta_{i}^{k}-2 g^{c a} g^{b j} \delta_{i}^{k}\right) D_{c a b} K_{j k} \\
& +2 g^{j[k} \delta^{l]}{ }_{i} \partial_{k} K_{j l}, \\
\mathcal{E}_{i}= & \partial_{i} Q^{-} T_{i}, \\
\mathcal{E}_{k}{ }^{i}= & N^{-1} \partial_{k} N^{i}-M_{k}^{i}, \\
\mathcal{E}_{k i j}= & \partial_{k} g_{i j}-2 D_{k i j}, \\
\mathcal{C}_{i j}= & 2 \partial_{[i} T_{j]}, \\
\mathcal{C}_{n k}{ }^{i}= & 2 \partial_{[n} M_{k]}{ }^{i}+2 M_{[k}^{i}\left(T_{n]}+2 \sigma D_{n] a b} g^{a b}\right), \\
\mathcal{C}_{k l i j}= & 2 \partial_{[k} D_{l] i j} .
\end{aligned}
$$

We note that these expressions do not contain any spatial derivatives of the metric fields $Q, N^{a}$ or $g_{i j}$ : these derivatives were replaced by $T_{i}, M_{k}{ }^{i}$ and $D_{k i j}$ wherever they occurred.

The collection of constraints,

$$
c^{\alpha}=\left\{\mathcal{C}, \mathcal{C}_{i}, \mathcal{E}_{i}, \mathcal{E}_{k}^{i}, \mathcal{E}_{k i j}, \mathcal{C}_{i j}, \mathcal{C}_{n k}^{i}, \mathcal{C}_{k l i j}\right\}
$$

is a function of the set of dynamical fields $\left\{g_{i j}, K_{i j}, D_{k i j}\right.$, $\left.Q, T_{i}, N^{i}, M_{k}^{i}\right\}$, hence the evolution of the constraints is determined by the dynamical evolution equations (3.18)(3.24). A straightforward (but very lengthy) calculation shows that the evolution of the constraints is determined by a system of the form

$$
\partial_{t} c^{\alpha}+C_{\beta}^{k \alpha}(u) \partial_{k} c^{\beta}=F_{\beta}^{\alpha}(u, \partial u) c^{\beta} .
$$

The coefficients $C^{k \alpha}{ }_{\beta}$ that appear in this expression depend on the metric fields $Q, N^{i}$, and $g_{i j}$ as well as the various parameters that enter Eqs. (3.18)-(3.24). The coefficients $F^{\alpha}{ }_{\beta}$ depend on all of the dynamical fields and their derivatives. The complete expressions for the constraint evolution equations are very lengthy, so here we list only the principal parts of the equations, $\partial_{t} c^{\alpha} \simeq-C^{k \alpha}{ }_{\beta}(u) \partial_{k} c^{\beta}$, which can be written

$$
\begin{aligned}
\partial_{t} \mathcal{C} \simeq & N^{n} \partial_{n} \mathcal{C}-\frac{1}{2} N(2+2 \chi-\eta) g^{i j} \partial_{i} \mathcal{C}_{j} \\
& +\frac{1}{4} N\left(2+2 \psi_{10}+3 \psi_{8}-4 \psi_{9}\right) g^{i j} \partial_{i} \mathcal{C}_{j k}{ }^{k},
\end{aligned}
$$

$$
\begin{aligned}
& \partial_{t} \mathcal{C}_{i} \simeq-N(1+2 \gamma) \partial_{i} \mathcal{C}+\frac{1}{2} N(1+\zeta) g^{j k} g^{a b} \partial_{j} \mathcal{C}_{a i k b} \\
& -\frac{1}{2} N g^{j k} \partial_{j} \mathcal{C}_{k i}-\frac{1}{2} N(1+2 \sigma) g^{j k} g^{a b} \partial_{j} \mathcal{C}_{k i a b} \\
& +N^{n} \partial_{n} \mathcal{C}_{i}+\frac{1}{2} N(1-\zeta) g^{j k} g^{a b} \partial_{j} \mathcal{C}_{k a b i} \\
& \partial_{t} \mathcal{E}_{i} \simeq 0 \\
& \partial_{t} \mathcal{E}_{k}^{i} \simeq 0 \text {, } \\
& \partial_{t} \mathcal{E}_{k i j} \simeq N^{n} \partial_{n} \mathcal{E}_{k i j}, \\
& \partial_{t} \mathcal{C}_{i j} \simeq N \psi_{1} \partial_{[i} \mathcal{C}_{j]}+\frac{1}{2} N \psi_{2} \partial_{k} \mathcal{C}_{i j}{ }^{k}, \\
& \partial_{t} \mathcal{C}_{n k}{ }^{i} \simeq N^{j} \partial_{j} \mathcal{C}_{n k}{ }^{i}+N \psi_{7} \delta_{[k}^{i} \partial_{n]} \mathcal{C}+N \psi_{3} g^{i j} \partial_{[n} \mathcal{C}_{k] j} \\
& -N \psi_{4} g^{i j} g^{a b} \partial_{[n} \mathcal{C}_{k] j a b}-N \psi_{5} g^{i j} g^{a b} \partial_{[n} \mathcal{C}_{k] a b j} \\
& +N \psi_{6} g^{i j} g^{a b} \partial_{[n} \mathcal{C}_{|j a b| k]}, \\
& \partial_{t} \mathcal{C}_{l k i j} \simeq N^{a} \partial_{a} \mathcal{C}_{l k i j}+N g_{a(i} \partial_{j)} \mathcal{C}_{l k}{ }^{a}+N \psi_{9} g_{i j} \partial_{[l} \mathcal{C}_{k] a}{ }^{a} \\
& +N \chi g_{i j} \partial_{[l} \mathcal{C}_{k]}-\frac{1}{2} N \eta\left(g_{i[l} \partial_{k]} \mathcal{C}_{j}+g_{j[l} \partial_{k]} \mathcal{C}_{i}\right) \\
& -\frac{1}{2} N \psi_{8}\left(g_{a i} \partial_{[l} \mathcal{C}_{k] j}{ }^{a}+g_{a j} \partial_{[l} \mathcal{C}_{k] i}{ }^{a}\right) \\
& -\frac{1}{2} N \psi_{10}\left(g_{i[l} \partial_{k]} \mathcal{C}_{j a}{ }^{a}+g_{j[l} \partial_{k]} \mathcal{C}_{i a}{ }^{a}\right) .
\end{aligned}
$$

We note that the principal parts of the constraint evolution equations can be written in a number of different ways using various identities that the constraints satisfy

$$
\begin{aligned}
\partial_{[i} \mathcal{E}_{j]}= & \frac{1}{2} \mathcal{C}_{j i}, \\
\partial_{[k} \mathcal{E}_{j]}{ }^{i}= & \frac{1}{2} \mathcal{C}_{j k}{ }^{i}+N^{-1} \mathcal{E}_{[k}{ }^{i} \partial_{j]} N+M_{[k}{ }^{i}\left(\mathcal{E}_{j]}+\sigma \mathcal{E}_{j] a b} g^{a b}\right), \\
\partial_{[l} \mathcal{E}_{k] i j}= & \mathcal{C}_{k l i j}, \\
\partial_{[k} \mathcal{C}_{i j]}= & 0, \\
\partial_{[n} \mathcal{C}_{j k]}{ }^{i}= & \mathcal{C}_{[k j}{ }^{i}\left(T_{n]}+2 \sigma D_{n] a b} g^{a b}\right) \\
& +M_{[n}{ }^{i}\left(\mathcal{C}_{j k]}+2 \sigma \mathcal{C}_{j k] a b} g^{a b}\right) \\
& +4 \sigma M_{[n}{ }^{i} \mathcal{E}_{k}{ }^{a b} D_{j] a b}, \\
\partial_{[n} \mathcal{C}_{k l] i j}= & 0 .
\end{aligned}
$$

We have not yet explored how the character of the constraint evolution equations (e.g., their hyperbolicity) is affected by changing their principal parts using these identities. 
We note that Eq. (A10) implies that the time derivative of the constraints vanishes, $\partial_{t} c^{\alpha}=0$, whenever the constraints are satisfied, $c^{\alpha}=0$, at some initial time. This guarantees that the constraints will be satisfied for all time in analytic spacetimes. And if these constraint evolution equations are symmetric hyperbolic (as we expect, but have not yet verified) then this will guarantee quite generally that the constraints remain satisfied for all time if they are satisfied initially.

\section{APPENDIX B: THE KST SYSTEM}

The fixed-gauge version of the Einstein evolution equations proposed by KST can be shown to be strongly or even symmetric hyperbolic for certain choices of the five "dynamical" free parameters $\{\sigma, \zeta, \gamma, \eta, \chi\}$ characterizing that system [12]. For the case in which all of the adjustable characteristic speeds are set equal to the speed of light, we have previously [13] determined the regions of this parameter space where the equations are symmetric hyperbolic, and the regions where they are not.

Using the technique developed in Sec. IV, we can now construct all of the symmetric hyperbolic fixed-gauge KST systems explicitly, even for the general case in which the adjustable speeds are left unspecified. For the fixed-gauge KST evolution system, Eqs. (3.1)-(3.3), the five dynamical parameters $\{\sigma, \zeta, \gamma, \eta, \chi\}$ can be written in terms of the symmetrizer parameters $\left\{B_{1}, C_{1}, C_{2}, C_{3}, C_{4}, C_{5}\right\}$. These conditions, which are equivalent to the conditions for symmetric hyperbolicity in these systems, are

$$
\begin{aligned}
B_{2} & =\left(C_{1}+2 C_{2}\right) / 3, \\
\zeta & =-3 C_{1} /\left(C_{1}+2 C_{2}\right),
\end{aligned}
$$

$$
\begin{aligned}
\gamma= & -\frac{32 C_{1}+10 C_{2}+45\left(C_{4}+C_{5}+2 B_{1}\right)}{135 B_{1}} \\
\sigma= & \frac{18 C_{1}+45\left(C_{3}+C_{4}+2 C_{5}\right)}{10\left(9 B_{1}+2 C_{1}+4 C_{2}\right)}, \\
\eta= & \frac{6}{5}+B_{2}\left[5\left(3 C_{3}+C_{4}+4 C_{5}\right)+20\left(C_{4}+3 C_{5}\right) \sigma\right. \\
& \left.-3\left(9 C_{3}+C_{4}+6 C_{5}\right) \zeta\right] / 25\left(C_{3} C_{4}-C_{5}^{2}\right), \\
\chi= & -\frac{2}{5}-B_{2}\left[5\left(C_{3}+2 C_{4}+3 C_{5}\right)+20\left(2 C_{4}+C_{5}\right) \sigma\right. \\
& \left.-3\left(3 C_{3}+2 C_{4}+7 C_{5}\right) \zeta\right] / 25\left(C_{3} C_{4}-C_{5}^{2}\right)
\end{aligned}
$$

Specifying the parameters $\{\sigma, \zeta, \gamma, \eta, \chi\}$ in this way guarantees that the characteristic speeds of this system,

$$
\begin{aligned}
& v_{1}^{2}=2 \sigma, \\
& v_{2}^{2}=\frac{1}{8} \eta(1-3 \zeta-4 \sigma)-\frac{1}{4} \chi(1+6 \sigma), \\
& v_{3}^{2}=\frac{1}{2}[(1+2 \gamma)(2+2 \chi-\eta)-\eta \zeta],
\end{aligned}
$$

are real, that the characteristic eigenvectors of the system are complete, etc. We also note that the expression for the parameters $\zeta$ and $\sigma$ in Eqs. (B2) and (B4), enforce the conditions that $\zeta$ and $\sigma$ be limited to the ranges $-3<\zeta<0$ and $0<\sigma$ for the symmetric hyperbolic fixed-gauge KST systems no matter what values the characteristic speeds may have.
[1] J.W. York, Jr., in Sources of Gravitational Radiation, edited by L.L. Smarr (Cambridge University Press, Cambridge, England, 1979), pp. 83-126.

[2] S. Frittelli and O. Reula, Commun. Math. Phys. 166, 221 (1994).

[3] Y. Choquet-Bruhat and J.W. York, Jr., C. R. Acad. Sci., Ser. I: Math. A321, 1089 (1995).

[4] H. Friedrich, Class. Quantum Grav. 13, 1451 (1996).

[5] S. Frittelli and O.A. Reula, Phys. Rev. Lett. 76, 4667 (1996).

[6] C. Bona, J. Massó, E. Seidel, and J. Stela, Phys. Rev. D 56, 3405 (1997).

[7] A. Anderson, Y. Choquet-Bruhat, and J.W. York, Jr., Topol. Methods Nonlinear Anal. 10, 353 (1997).

[8] A. Anderson and J.W. York, Jr., Phys. Rev. Lett. 82, 4384 (1999).

[9] S. Frittelli and O.A. Reula, J. Math. Phys. 40, 5143 (1999).

[10] M. Alcubierre, B. Brügmann, M. Miller, and W.-M. Suen, Phys. Rev. D 60, 064017 (1999).

[11] S.D. Hern, Ph.D. thesis, University of Cambridge, 1999, gr-qc/0004036.

[12] L.E. Kidder, M.A. Scheel, and S.A. Teukolsky, Phys. Rev. D 64, 064017 (2001).

[13] L. Lindblom and M.A. Scheel, Phys. Rev. D 66, 084014 (2002)
[14] L.L. Smarr, in Eighth Texas Symposium on Relativistic Astrophysics, edited by M.D. Papagiannis (The New York Academy of Sciences, New York, 1977), pp. 569-604.

[15] L.I. Petrich, S.L. Shapiro, and S.A. Teukolsky, Phys. Rev. D 31, 2459 (1985).

[16] M.W. Choptuik, E.W. Hirschmann, S.L. Liebling, and F. Pretorius, Class. Quantum Grav. 20, 1857 (2003).

[17] A.E. Fischer and J.E. Marsden, in General Relativity: An Einstein Centenary Survey, edited by S.W. Hawking and W. Israel (Cambridge University Press, Cambridge, England, 1979), pp. $138-211$.

[18] L.L. Smarr and J.W. York, Jr., Phys. Rev. D 17, 2529 (1978).

[19] M. Alcubierre, Class. Quantum Grav. 20, 607 (2003).

[20] M. Alcubierre, B. Brügmann, D. Pollney, E. Seidel, and R. Takahashi, Phys. Rev. D 64, 061501(R) (2001).

[21] M. Alcubierre, B. Brügmann, P. Diener, M. Koppitz, D. Pollney, E. Seidel, and R. Takahashi, Phys. Rev. D 67, 084023 (2003).

[22] H.-J. Yo, T.W. Baumgarte, and S.L. Shapiro, Phys. Rev. D 66, 084026 (2002).

[23] D. Shoemaker, K. Smith, E. Schnetter, D. Fiske, and P. Laguna (in preparation).

[24] K. Alvi, Class. Quantum Grav. 19, 5153 (2002). 
[25] C. Bona, J. Stela, J. Masso, and E. Seidel, in Proceedings of the Seventh Marcel Grossmann Meeting on General Relativity, edited by R.T. Jantzen, G.M. Keiser, and R. Ruffini (World Scientific, Singapore, 1996), pp. 410-412.

[26] C. Bona, J. Massó, E. Seidel, and J. Stela, Phys. Rev. Lett. 75, 600 (1995).

[27] O. Sarbach and M. Tiglio, Phys. Rev. D 66, 064023 (2002).

[28] M. Alcubierre and B. Brügmann, Phys. Rev. D 63, 104006 (2001).

[29] E. Seidel and W.-M. Suen, Phys. Rev. Lett. 69, 1845 (1992).

[30] G.B. Cook et al., Phys. Rev. Lett. 80, 2512 (1998).

[31] S. Brandt et al., Phys. Rev. Lett. 85, 5496 (2000).

[32] M.A. Scheel, L.E. Kidder, L. Lindblom, H.P. Pfeiffer, and S.A. Teukolsky, Phys. Rev. D 66, 124005 (2002).

[33] D. Shoemaker, K. Smith, U. Sperhake, P. Laguna, E. Schnetter, and D. Fiske, gr-qc/0301111.

[34] G.B. Cook, Phys. Rev. D 65, 084003 (2002).

[35] The factors of the lapse $N$ that appear in this and subsequent equations were chosen to make these wave equations more covariant. Thus for example by using the coefficient $\mu N^{2}$ instead of $\mu$ in Eq. (2.3) the characteristic speeds relative to a surface normal observer are $\pm \sqrt{\mu}$ instead of $\pm \sqrt{\mu} / N$.

[36] J. Balakrishna, G. Daues, E. Seidel, W.-M. Suen, M. Tobias, and E. Wang, Class. Quantum Grav. 13, L135 (1996).

[37] M. Holst (unpublished).

[38] M. Shibata and T. Nakamura, Phys. Rev. D 52, 5428 (1995).

[39] T.W. Baumgarte and S.L. Shapiro, Phys. Rev. D 59, 024007 (1999).

[40] Note that we can change the principal parts of Eqs. (3.6) and (3.7) by adding terms proportional to $N^{k}\left(\partial_{k} Q-T_{k}\right)$ and $N^{k}\left(\partial_{k} N^{i}-N M_{k}^{i}\right)$. However, doing this for the shift equation spoils linear degeneracy, making the system genuinely nonlinear. We chose to keep the system linearly degenerate since this condition is known to prevent the formation of shocks in similar (but not identical) hyperbolic systems [51].

[41] We note that the 3-index constraint $\mathcal{C}_{n k}{ }^{i}$ used in this paper is not the same as the 3 -index constraint $\mathcal{C}_{k i j}$ introduced by KST [12].

[42] We note that the constraints are to be thought of as functions of $Q, N^{i}, g_{i j}, K_{i j}, D_{k i j}, T_{i}, M_{k}{ }^{i}$ and the first derivatives $\partial_{k} K_{i j}$, $\partial_{l} D_{k i j}, \partial_{k} T_{i}$, and $\partial_{l} M_{k}{ }^{i}$. The explicit expressions for the constraints in terms of these quantities are given in Appendix A.

[43] R. Courant and D. Hilbert, Methods of Mathematical Physics
(Wiley, New York, 1962), Vol. II.

[44] We have considered the most general symmetrizer that can be constructed from the spatial metric and constant parameters. The symmetrizer given in Eq. (4.2), however, lacks a number of cross terms between the metric variables $\left\{g_{i j}, Q, N^{i}\right\}$ and the derivative variables $\left\{K_{i j}, D_{k i j}, T_{i}, M_{k}{ }^{i}\right\}$. These cross terms must vanish as a consequence of the symmetry conditions, and so for simplicity we omit them from Eq. (4.2).

[45] W.H. Press, S.A. Teukolsky, W.T. Vetterling, and B.P. Flannery, Numerical Recipes in C, 2nd ed. (Cambridge University Press, Cambridge, England, 1992).

[46] We note that Eqs. (4.13)-(4.32) are ordered so that each of the parameters $\left\{B_{2}, \psi_{1}, \ldots, \psi_{10}, \sigma, \gamma, \eta, \chi, \zeta, \mu_{L}, \mu_{S}, \epsilon_{L}\right.$, $\left.\epsilon_{S}\right\}$ appears on the right-hand side of only those equations that follow the one in which it appears on the left.

[47] The characteristic speeds associated with these fields are actually quite arbitrary. For example, we could change them to arbitrary multiples of $-\xi_{k} N^{k} / N$ (including zero) simply by adding the terms $V^{k}\left(\partial_{k} Q-T_{k}\right)$ and $V^{k}\left(\partial_{k} N^{i}-N M_{k}{ }^{i}\right)$ to Eqs. (3.19) and (3.20) with $V^{k}$ an arbitrary multiple of $N^{k}$. Terms of this type do not change the symmetric hyperbolicity of the system, but they can affect its linear degeneracy (and hence the ability of the system to form shocks). The vector $V^{k}$ that appears in these extra terms could also be specified as an arbitrary function of the coordinates. In this case these characteristic speeds become $-\xi_{k}\left(N^{k}-V^{k}\right) / N$ which can be adjusted to arbitrary values with appropriate choice of $V^{k}$ while leaving the system linearly degenerate. These speeds can all be made less than the speed of light in this way if that is desired.

[48] Y. Choquet-Bruhat and J.W. York, Jr., Banach Cent Publ. 41, 119 (1997).

[49] The characteristic speeds associated with the fields $Q$ and $N^{i}$ are $-\xi_{i} N^{i} / N$ and cannot be changed by adjusting the various parameters of the theory. These speeds will be less than the speed of light except when the vector $\partial_{t}$ becomes spacelike. However, as previously noted the equations can be easily modified to make these speeds causal if that is desired.

[50] This is an extension (with a slight change in notation) of the transformation introduced by KST [12]. The relationship between the original KST notation and that used here is $\hat{z}_{1}=\hat{z}$, $\hat{k}_{1}=\hat{k}, \hat{k}_{2}=\hat{e}, \hat{k}_{3}=\hat{a}, \hat{k}_{4}=\hat{b}, \hat{k}_{5}=\hat{c}$, and $\hat{k}_{6}=\hat{d}$.

[51] T.P. Liu, J. Diff. Eqns. 33, 92 (1979). 